$\underline{\text { Preprint typeset in JHEP style - HYPER VERSION }}$

hep-th/0702150

TIFR/TH/06-32

\title{
Comments on the Spectrum of CHL Dyons
}

\author{
Atish Dabholkar*1, ${ }^{*}$, Davide Gaiotto ${ }^{\dagger 3}$, and Suresh Nampuri ${ }^{\ddagger 1}$ \\ ${ }^{1}$ Department of Theoretical Physics \\ Tata Institute of Fundamental Research \\ Homi Bhabha Rd, Mumbai 400 005, India \\ ${ }^{2}$ Laboratoire de Physique Théorique et Hautes Energies (LPTHE) \\ Tour 24-25, 5ème étage, Boite 126, 4 Place Jussieu, 75252 Paris Cedex 05 \\ Unité Mixte de Recherche (UMR 7589) \\ Université Pierre et Marie Curie-Paris 6; CNRS; Université Denis Diderot-Paris 7 \\ ${ }^{3}$ Jefferson Labs, \\ 17 Oxford St, Harvard University \\ Cambridge, MA 02138 USA
}

\begin{abstract}
We address a number of puzzles relating to the proposed formulae for the degeneracies of dyons in orbifold compactifications of the heterotic string to four dimensions with $\mathcal{N}=4$ supersymmetry. The partition function for these dyons is given in terms of Siegel modular forms associated with genus-two Riemann surfaces. We point out a subtlety in demonstrating S-duality invariance of the resulting degeneracies and give a prescription that makes the invariance manifest. We show, using M-theory lift of string webs, that the genus-two contribution captures the degeneracy only if a specific irreducibility criterion is satisfied by the charges. Otherwise, in general there can be additional contributions from higher genus Riemann surfaces. We analyze the negative discriminant states predicted by the formula. We show that even though there are no big black holes in supergravity corresponding to these states, there are multi-centered particle-like configurations with subleading entropy in agreement with the microscopic prediction and our prescription for S-duality invariance. The existence of the states is moduli dependent and we exhibit the curves of marginal stability and comment on its relation to S-duality invariance.
\end{abstract}

*Email: atish@theory.tifr.res.in

†Email: dgaiotto@fas.harvard.edu

†Email: suresh@theory.tifr.res.in 


\section{Contents}

1. Introduction 1

2. The Dyon Partition Function 2

3. S-Duality Invariance 4

4. Irreducibility Criterion and Higher Genus Contributions 7

5. States with Negative Discriminant 11

5.1 Microscopic Prediction 13

5.2 Supergravity Analysis 14

5.3 Moduli Dependence and Lines of Marginal Stability 17

6. Interpretation and Discussion 20

\section{Introduction}

The spectrum of BPS states has played a very significant role in several important developments in string theory. In the limit of small charges, counting low-lying BPS states has provided many nontrivial tests of duality. In the opposite limit of large charges, computation of asymptotic degeneracies of BPS states has allowed for a statistical interpretation of the Bekenstein-Hawking entropy of certain supersymmetric black holes addressing a long-standing problem in quantum gravity. It is clearly desirable to obtain similar quantitative information about the spectrum of BPS states with arbitrary intermediate charges.

Many BPS states can be mapped by duality to either perturbative winding-momentum states or a collection of D-branes in a particular duality frame. In both cases, using powerful techniques from the worldsheet and from gauge theory, it is possible to obtain detailed exact information about the BPS spectrum for all values of charges. To complete the picture, one would like to develop tools to analyze the spectrum of BPS states that are nonperturbative in all duality frames. Such states, for example various dyons in four dimensions, are in a sense more interesting because they can allow us to access the interior regions of moduli space at intermediate couplings that are not weakly coupled in any duality frame. 
For heterotic string compactifications with $\mathcal{N}=4$ supersymmetry in four dimensions, there exists a formula for the exact degeneracies of the dyonic quarter-BPS states in terms of Siegel modular forms of $S p(2, \mathbb{Z})$ and its subgroups [1, 2]. As for the perturbative winding-momentum states or for D-brane bound states, it is desirable to have a systematic derivation of the dyon partition function using worldsheet or gauge theory techniques. A weak coupling derivation has been suggested recently using the $4 \mathrm{~d}-5 \mathrm{~d}$ lift [3, 4]. A similar derivation for the more general orbifolds with $\mathcal{N}=4$ supersymmetry is discussed in [5, 6, 7, 8]. The role of genus-two Riemann surfaces and the appearance of $\operatorname{Sp}(2, \mathbb{Z})$ can be explained using an M-theory lift of string webs [9] and an alternative derivation of the dyon partition function in terms of a genus-two partition function of the heterotic string has been given in [5, 10.

Our purpose here is to address a number of subtleties in the interpretation and derivation of the dyon partition function using these ideas. We first summarize the basic ingredients of the dyon partition function in $\S 2$. In $\S 3$ we discuss the S-duality invariance and give a prescription for the choice of the contours that yields manifestly duality invariant spectrum. In $\S \varangle$, using M-theory lift of string webs, we show that the genus-two formula is adequate only if the charges satisfy a specific irreducibility criterion. Otherwise there can be additional contributions from higher genus Riemann surfaces. In $\$ 5$ we consider states predicted by the formula that have negative discriminant for which there are no big black holes in supergravity corresponding to these states. We discuss a simple example for such states with negative discriminant in $\$ 5.1$, and show that there are multi-centered particle-like configurations with subleading entropy in agreement with the microscopic prediction as well as with our prescription for S-duality invariance. The supergravity solutions analyzed in $\$ 5.2$ display an intricate moduli dependence. We show in $\$ 5.3$ that a two-centered solution with the desired degeneracy exists in a large region of moduli space where supergravity and string loop expansion is under control. However, there a straight line in the axion dilaton space defines a line of marginal stability. The state exists only on one side of the line, decays into two fragments as one approaches this line, and ceases to exist on the other side of the line. We conclude in $\$ 6$ with a discussion of the interpretation of the dyon degeneracy formula in the light of above-mentioned considerations of irreducibility criterion, moduli dependence, lines of marginal stability, and duality invariance.

\section{The Dyon Partition Function}

Let $\Omega$ be a $(2 \times 2)$ symmetric matrix with complex entries

$$
\Omega=\left(\begin{array}{ll}
\rho & v \\
v & \sigma
\end{array}\right)
$$

satisfying

$$
(\operatorname{Im} \rho)>0, \quad(\operatorname{Im} \sigma)>0, \quad(\operatorname{Im} \rho)(\operatorname{Im} \sigma)>(\operatorname{Im} v)^{2}
$$


which parametrizes the 'Siegel upper half plane' in the space of $(\rho, v, \sigma)$. It can be thought of as the period matrix of a genus two Riemann surface. For a genus-two Riemann surface, there is a natural symplectic action of $S p(2, \mathbb{Z})$ on the period matrix. We write an element $g$ of $S p(2, \mathbb{Z})$ as a $(4 \times 4)$ matrix in the block form as

$$
\left(\begin{array}{ll}
A & B \\
C & D
\end{array}\right)
$$

where $A, B, C, D$ are all $(2 \times 2)$ matrices with integer entries. They satisfy

$$
A B^{T}=B A^{T}, \quad C D^{T}=D C^{T}, \quad A D^{T}-B C^{T}=I,
$$

so that $g^{t} J g=J$ where $J=\left(\begin{array}{cc}0 & -I \\ I & 0\end{array}\right)$ is the symplectic form. The action of $g$ on the period matrix is then given by

$$
\Omega \rightarrow(A \Omega+B)(C \Omega+D)^{-1} .
$$

The object of our interest is a Siegel modular form $\Phi_{k}(\Omega)$ of weight $k$ which transforms as

$$
\Phi_{k}\left[(A \Omega+B)(C \Omega+D)^{-1}\right]=\{\operatorname{det}(C \Omega+D)\}^{k} \Phi_{k}(\Omega)
$$

under an appropriate congruence subgroup of $S p(2, \mathbb{Z})[2]$. The subgroup as well as the index $k$ of the modular form are determined in terms of the order $N$ of the particular CHL $\mathbb{Z}_{N}$ orbifold one is considering [2]. In a given CHL model, the inverse of the $\Phi_{k}$ is to be interpreted then as a partition function of dyons.

To see in more detail how the dyon degeneracies are defined in terms of the partition function, let us consider for concreteness the simplest model of toroidally compactified heterotic string as in the original proposal of Dijkgraaf, Verlinde, Verlinde [1]. Many of the considerations extend easily to the more general orbifolds with $\mathcal{N}=4$ supersymmetry. In this case the relevant modular form is the well-known Igusa cusp form $\Phi_{10}(\Omega)$ of weight ten of the full group $\operatorname{Sp}(2, \mathbb{Z})$. A dyonic state is specified by the charge vector $Q=\left(Q_{e}, Q_{m}\right)$ which transforms as a doublet of the S-duality group $S L(2, \mathbb{R})$ and as a vector of the T-duality group $O(22,6 ; \mathbb{Z})$. There are three T-duality invariant quadratic combinations $Q_{m}^{2}, Q_{e}^{2}$, and $Q_{e} \cdot Q_{m}$ that one can construct from these charges. Given these three combinations, the degeneracy $d(Q)$ of dyonic states of charge $Q$ is then given by

$$
d(Q)=g\left(\frac{1}{2} Q_{m}^{2}, \frac{1}{2} Q_{e}^{2}, Q_{e} \cdot Q_{m}\right)
$$

where $g(m, n, l)$ are the Fourier coefficients of $1 / \Phi_{10}$,

$$
\frac{1}{\Phi_{10}(\rho, \sigma, v)}=\sum_{m \geq-1, n \geq-1, l} e^{2 \pi i(m \rho+n \sigma+l v)} g(m, n, l) .
$$


The parameters $(\rho, \sigma, v)$ can be thought of as the chemical potentials conjugate to the integers $\left(\frac{1}{2} Q_{m}^{2}, \frac{1}{2} Q_{e}^{2}, Q_{e} \cdot Q_{m}\right)$ respectively. The degeneracy $d(Q)$ obtained this way satisfies a number of physical consistency checks. For large charges, its logarithm agrees with the BekensteinHawking-Wald entropy of the corresponding black holes to leading and the first subleading order 11, 11, 2, 12, 13. It is integral as expected for an object that counts the number of states. It is formally S-duality invariant [1, 2] but as we will see in the next section the formal proof is not adequate. An appropriate prescription is necessary as we explain in detail in the next section which also allows for a nontrivial moduli dependence.

\section{S-Duality Invariance}

The first physical requirement on the degeneracy $d(Q)$ given by $(2.7)$ is that it should be invariant under the S-duality group of the theory. For the simplest case of toroidal compactification that we are considering, the S-duality group is $S L(2, \mathbb{Z})$ and more generally for $\mathbb{Z}_{N}$ CHL orbifolds its a congruence subgroup $\Gamma_{1}(N)$ of $S L(2, \mathbb{Z})$. So, we would like to show for the $N=1$ example, that the degeneracy (2.7) is invariant under an S-duality transformation

$$
Q_{e} \rightarrow Q_{e}^{\prime}=a Q_{e}+b Q_{m}, \quad Q_{m} \rightarrow Q_{m}^{\prime}=c Q_{e}+d Q_{m}, \quad\left(\begin{array}{ll}
a & b \\
c & d
\end{array}\right) \in S L(2, \mathbb{Z}) .
$$

A formal proof of S-duality following [1, 2] proceeds as follows. Inverting the relation (2.8) we can write

$$
d(Q)=\int_{\mathcal{C}} d^{3} \Omega e^{-i \pi Q^{\prime t} \cdot \Omega \cdot Q} \frac{1}{\Phi_{10}(\Omega)}
$$

where the integral is over the contours

$$
0<\rho \leq 1, \quad 0<\sigma \leq 1, \quad 0<v \leq 1
$$

along the real axes of the three coordinates $(\rho, \sigma, v)$. This defines the integration curve $\mathcal{C}$ as a 3 -torus in the Siegel upper half plane. Now we would like to show

$$
d\left(Q^{\prime}\right)=\int_{\mathcal{C}} d^{3} \Omega e^{-i \pi Q^{\prime t} \cdot \Omega \cdot Q^{\prime}} \frac{1}{\Phi_{10}(\Omega)}
$$

equals $d(Q)$. To do so, we define

$$
\Omega^{\prime} \equiv\left(\begin{array}{ll}
\rho^{\prime} & v^{\prime} \\
v^{\prime} & \sigma^{\prime}
\end{array}\right)=(A \Omega+B)(C \Omega+D)^{-1}
$$

for

$$
\left(\begin{array}{ll}
A & B \\
C & D
\end{array}\right)=\left(\begin{array}{cccc}
a & -b & b & 0 \\
-c & d & 0 & c \\
0 & 0 & d & c \\
0 & 0 & b & a
\end{array}\right) \in S p(2, \mathbb{Z})
$$


We can change the integration variable from $\Omega$ to $\Omega^{\prime}$. Using these transformation properties and the modular properties of $\Phi_{10}$ we see that

$$
\begin{aligned}
d^{3} \Omega^{\prime} & =d^{3} \Omega, \\
\Phi_{10}\left(\Omega^{\prime}\right) & =\Phi_{10}(\Omega), \\
Q^{\prime t} \cdot \Omega^{\prime} \cdot Q^{\prime} & =Q^{t} \cdot \Omega \cdot Q
\end{aligned}
$$

Moreover, the integration contour $\mathcal{C}$ as defined in $(3.3)$ is invariant under the duality transformation on the integration variables (3.5). We therefore conclude

$$
d\left(Q^{\prime}\right)=\int_{\mathcal{C}} d^{3} \Omega^{\prime} e^{-i \pi Q^{\prime T} \cdot \Omega^{\prime} Q^{\prime}} \frac{1}{\Phi_{10}\left(\Omega^{\prime}\right)}=d(Q) .
$$

This formal proof is however not quite correct. The reason is that the partition function $1 / \Phi_{10}$ has a double pole at $v=0$ which lies on the integration contour $\mathcal{C}$. Thus the integral in (3.2) is not well-defined on the contour $\mathcal{C}$ and one must give an appropriate prescription for the integration. The non-invariance can also be seen explicitly from the Fourier expansion. To illustrate the point, let us look at states with

$$
\frac{1}{2} Q_{m}^{2}=-1, \quad \frac{1}{2} Q_{e}^{2}=-1, \quad Q_{e} \cdot Q_{m}=N .
$$

Then according to (2.7), the degeneracy of such states can be read off from the coefficient of $y^{N} / q p$ in the Fourier expansion (2.8). From the product representation of $\Phi_{10}$ given for example in [1] , we see that we need to pick the term that goes as $p^{-1} q^{-1} y^{N}$ in the expansion of

$$
\frac{1}{q p\left(y^{\frac{1}{2}}-y^{-\frac{1}{2}}\right)^{2}}=\frac{y}{q p} \frac{1}{(1-y)^{2}}=\sum_{N=1}^{\infty} N q^{-1} p^{-1} y^{N}
$$

which implies that

$$
d(-1,-1, N)=N
$$

Let us now look at what is required for invariance under $S L(2, \mathbb{Z})$ transformations. Consider, for example, the element

$$
S=\left(\begin{array}{cc}
0 & 1 \\
-1 & 0
\end{array}\right)
$$

of the S-duality group which takes $\left(Q_{e}, Q_{m}\right)$ to $\left(Q_{m},-Q_{e}\right)$. Hence $\left(\frac{1}{2} Q_{m}^{2}, \frac{1}{2} Q_{e}^{2}, Q_{e} \cdot Q_{m}\right)$ goes to $\left(\frac{1}{2} Q_{e}^{2}, \frac{1}{2} Q_{m}^{2},-Q_{e} \cdot Q_{m}\right)$. Invariance of the spectrum under this element of the S-duality group would then predict $d(-1,-1,-N)=d(-1,-1, N)=N$. However, from the expansion (3.12) we see that there are no terms in the Laurent expansion that go as $y^{-N}$ and hence an application of the formulae (2.7) and (2.8) would give $d(-1,-1,-N)=0$ in contradiction with the prediction from S-duality. 
This apparent lack of S-duality invariance is easy to fix with a more precise prescription. Note that the function $\left(y^{\frac{1}{2}}-y^{-\frac{1}{2}}\right)^{-2}$ has a $\mathbb{Z}_{2}$ symmetry generated by the element $S$ of the S-duality group that takes $y$ to $y^{-1}$. Under this transformation the contour $|y|<1$ is not left invariant but instead gets mapped to the contour $|y|>1$. The new contour cannot be deformed to the original one without crossing the pole at $y=1$ so if we are closing the contour around $y=0$ then we need to take into account the contribution from this pole at $y=1$. Alternatively, it is convenient to close the contour at $y^{-1}=0$ instead of $y=0$. Then we do not encounter any other pole and because of the symmetry of the function $\left(y^{\frac{1}{2}}-y^{-\frac{1}{2}}\right)^{-2}$ under $y$ going to $y^{-1}$, the Laurent expansion around $y$ has the same coefficients as the Laurent expansion around $y^{-1}$. We then get,

$$
\frac{1}{p q\left(y^{\frac{1}{2}}-y^{-\frac{1}{2}}\right)^{2}}=\frac{y^{-1}}{p q} \frac{1}{\left(1-y^{-1}\right)^{2}}=\sum_{N=1}^{\infty} N p^{-1} q^{-1} y^{-N} .
$$

If we now define $d(-1,-1,-N)$ as the coefficient of $q p y^{-N}$ in the expansion (3.15) instead of in the expansion (3.12) then $d(-1,-1,-N)=N=d(-1,-1, N)$ consistent with S-duality.

States with negative $N$ must exist if states with positive $N$ exist not only to satisfy Sduality invariance but also to satisfy parity invariance. The $\mathcal{N}=4$ super Yang-Mills theory is parity invariant. Under parity, our state with positive $N$ goes to a state with negative $N$ and the asymptotic values $\chi$ of the axion also flips sign at the same time. Hence if a state with $N$ positive exists at $\chi=\chi_{0}$ then a state with $N$ negative must exist at $\chi=-\chi_{0}$. Thus, the naive expansion (3.12) would give an answer inconsistent with parity invariance and one must use the prescription we have proposed, to satisfy parity invariance. Note that even though S-duality and parity both take the states $(-1,-1, N)$ to $(-1,-1,-N)$ they act differently on the moduli fields.

In either case, the important point is that to extract the degeneracies in an S-duality invariant way, we need to use different contours for different charges. The function $1 / \Phi_{10}$ has many more poles in the $(\rho, \sigma, v)$ space at various divisors that are the $S p(2, \mathbb{Z})$ images of the pole at $y=1$ and in going from one contour to the other these poles will contribute. Instead of specifying contours, a more practical way to state the prescription is to define the degeneracies $d(Q)$ by formulae (2.7) and (2.8) first for charges that belong to the 'fundamental cell' in the charge lattice satisfying the condition $\frac{1}{2} Q_{m}^{2} \geq-1, \frac{1}{2} Q_{e}^{2} \geq-1$, and $Q_{e} \cdot Q_{m} \geq 0$. For these charges $d(Q)$ can be represented as a contour integral for a contour of integration around $p=q=y=0$ that avoids all poles arising as images of $y=1$. This can be achieved by allowing $(\rho, v, \sigma)$ to all have a large positive imaginary part as noted also in [7]. For other charges, the degeneracy is defined by requiring invariance under $S L(2, \mathbb{Z})$. The degeneracies so defined are manifestly S-duality invariant. This statement of S-duality invariance might appear tautologous, but its consistency depends on the highly nontrivial fact that an analytic function defined by $\Phi_{10}(\rho, \sigma, v)$ exists that is $S L(2, \mathbb{Z})$ invariant. Its pole structure guarantees that one 
gets the same answer independent of which way the contour is closed.

The choice of integration contour is possibly related to moduli dependence of the spectrum. To see this let us understand in some detail what precisely is required for S-duality. Given a state with charge $Q$ that exists for the values of the moduli $\varphi$, the statement of S-duality only requires that the degeneracy $d(Q)$ at $\varphi$ be the same as the degeneracy $d\left(Q^{\prime}\right)$ at $\varphi^{\prime}$ where $Q^{\prime}$ and $\varphi^{\prime}$ are S-duality transforms of $Q$ and $\varphi$ respectively. In many cases, one can then invoke the BPS property to assume that as we move around in the moduli space, barring phase transitions, the spectrum can be analytically continued from $\varphi^{\prime}$ to $\varphi$ to deduce $d\left(Q^{\prime}\right)=d(Q)$ at $\varphi$. This argument is known to work perfectly for half-BPS states in theories with $\mathcal{N}=4$ supersymmetric but with lower supersymmetry or for quarter-BPS states in $\mathcal{N}=4$, generically there can be curves of marginal stability. In such cases, states with charges $Q^{\prime}$ may exist for moduli values $\varphi^{\prime}$ but not for $\varphi$ and similarly states with charges $Q$ may exist for moduli values $\varphi$ but not for $\varphi^{\prime}$. Therefore, there are two possibilities for extracting the dyon degeneracies.

- There are no curves of marginal stability in the dilaton-axion moduli space. In this case if two charge configurations $Q$ and $Q^{\prime}$ are related by S-duality, then $d(Q)=d\left(Q^{\prime}\right)$.

- There are curves of marginal stability in the dilaton-axion moduli space. In this case one can say at most that $d(Q)$ at $\varphi$ equals $d\left(Q^{\prime}\right)$ at $\varphi^{\prime}$.

We will return in $\$ 6$ to a further discussion of these possibilities in the present context after considering explicit examples of moduli dependence and lines of marginal stability in $\S$.

\section{Irreducibility Criterion and Higher Genus Contributions}

One way to derive the dyon partition function is to use the representation of dyons as string webs wrapping the $\mathbf{T}^{\mathbf{2}}$ factor in Type-IIB on $\mathbf{K} \mathbf{3} \times \mathbf{T}^{\mathbf{2}}$. Using M-theory lift, the partition function that counts the holomorphic fluctuations of this web can be related to the genus-two partition function of the left-moving heterotic string [9, 5, 10]. The appearance of genus-two is thus explained by the topology of the string web. Such a derivation immediately raises the possibility of contribution from higher genus Riemann surface because string webs with more complicated topology are certainly possible. In this section we address this question and show that the genus-two partition function correctly captures the dyon degeneracies if the charges satisfy certain irreducibility criteria. Otherwise, there are higher genus corrections to the genus-two formula.

There are various derivations of the dyon degeneracy formula, but often they compute the degeneracies for a specific subset of charges, and then use duality invariance to extend the result to generic charges. Such an application of duality invariance assumes in particular that under the duality group $S O(22,6, \mathbb{Z})$ the only invariants built out of charges would be $Q_{e}^{2}, Q_{m}^{2}$, and 


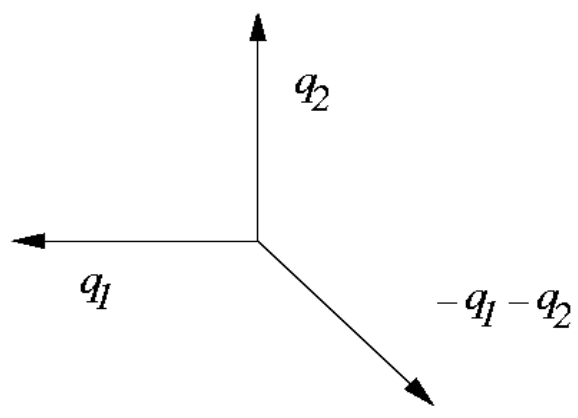

Figure 1: Charge conservation at a string junction

$Q_{e} \cdot Q_{m}$. This assumption is incorrect. If two charges are in the same orbit of the duality group, then obviously they have the same value for these three invariants. However the converse is not true. In general, for arithmetic groups, there can be discrete invariants which cannot be written as invariants of the continuous group.

An example of a non-trivial invariant that can be built out of two integral charge vectors is $I=\operatorname{gcd}\left(Q_{e} \wedge Q_{m}\right)$, i.e., the gcd of all bilinears $Q_{e}^{i} Q_{m}^{j}-Q_{e}^{j} Q_{m}^{i}$. Our goal is to show that the genus-two dyon partition function correctly captures the degeneracies if $I=1$. Note that half-BPS states have $I=0$ and hence are naturally associated with a genus-one surface. If $I>1$, then there are additional zero modes for the dyon under consideration and it would be necessary to correctly take them into account for counting the dyons.

The essential idea is to represent quarter-BPS states in the Type-IIB frame as a periodic string network wrapped on the two-torus. Type-IIB compactified on a $\mathbf{K} \mathbf{3}$ has a variety of half-BPS strings that can carry a generic set of $(21,5)$ charges arising from D5 and NS5 branes wrapped on the K3, D3-branes wrapped on some of the $(19,3)$ two-cycles as well as D1 and F1-strings. Several half-BPS strings can join into a web that preserve a quarter of the supersymmetries [14, 15, 16, 17]. The supersymmetry condition requires that the strings lie in a plane, and that their central charge vectors also lie in a plane. The strands must be oriented at relative angles that mimic the relative angle of their central charge vectors. The condition on the angles between strings guarantees the balance of tensions at the junction of three strands of the web as shown in Fig.1.

The central charges are given in terms of the charges and the scalar moduli of the theory as $Z=T q$. The matrix $T$ contains the scalar moduli of the theory, that parameterize the way a vector in the $\Gamma^{(21,5)}$ Narain lattice of charges decomposes into a left-moving and a right-moving part. The five-dimensional right-moving part is the vector of central charges for the string. For 


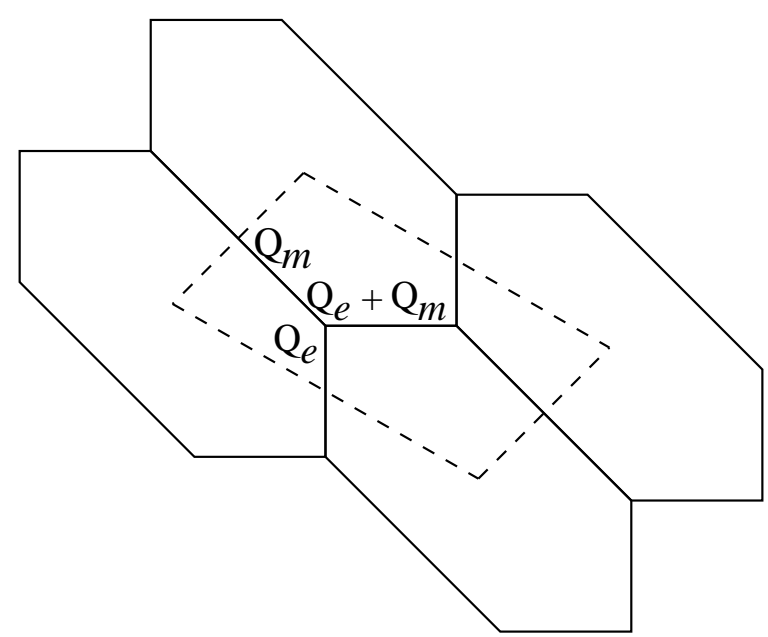

Figure 2: A quarter-BPS dyon carrying irreducible charges $Q_{e}$ and $Q_{m}$ with $\operatorname{gcd}\left(Q_{e} \wedge Q_{m}\right)=1$

generic values of the scalar moduli, one does not expect to have tensionless strings. Hence it follows that $T Q=0$ implies $Q=0$. The condition that all central charges $T Q_{i}$ should lie in a plane, $T Q_{i}=a_{i} T Q_{1}+b_{i} T Q_{2}$ is then equivalent to $Q_{i}=a_{i} Q_{1}+b_{i} Q_{2}$, i.e., the charge vectors $Q_{i}$ of all strings should also lie in a plane. A periodic string web can be wrapped on the torus of a $\mathbf{K} \mathbf{3} \times \mathbf{T}^{\mathbf{2}}$ compactification as shown in Fig. 2 .

After compactification on the torus, the strands of the web can carry additional charges: momentum along the direction they wrap, and KK monopole charge for the circle they do not wrap. The charges are organized in a $(22,6)$ charge vector. The result is a quarter-BPS dyon in the four dimensional theory. A dyon with generic charges $Q_{e}, Q_{m}$ typically has a very simple realization as a web with three strands. A simple possible choice of charges on the strands would be $Q_{e}, Q_{m}, Q_{e}+Q_{m}$. This web comes from the periodic identification of a hexagonal lattice. As the shape of the $T^{2}$ or the moduli in $T$ change, the size of one strand may become zero, and the web may degenerate into two cycles of the torus meeting at a point. On the other side, of the transition the intersection will open up in the opposite way and the configuration then smoothly become a new three-strands web. For example, the web with strands $Q_{e}, Q_{m}, Q_{e}+Q_{m}$ may go to a web with strands $Q_{e}, Q_{m}, Q_{e}-Q_{m}$. This process has interesting consequences on the stability of certain BPS states, that will be reviewed in 8 .

It has been argued [9] that the partition function of supersymmetric ground states for such webs can be computed by an unconventional lift to M-theory that relates it to a chiral genustwo partition function of the heterotic string. The genus-two partition function computed using this lift for CHL-orbifolds [5, 10] indeed equals the dyon partition function obtained by other means.

A priori, the string junction need not to be stable against opening up into more complicated 


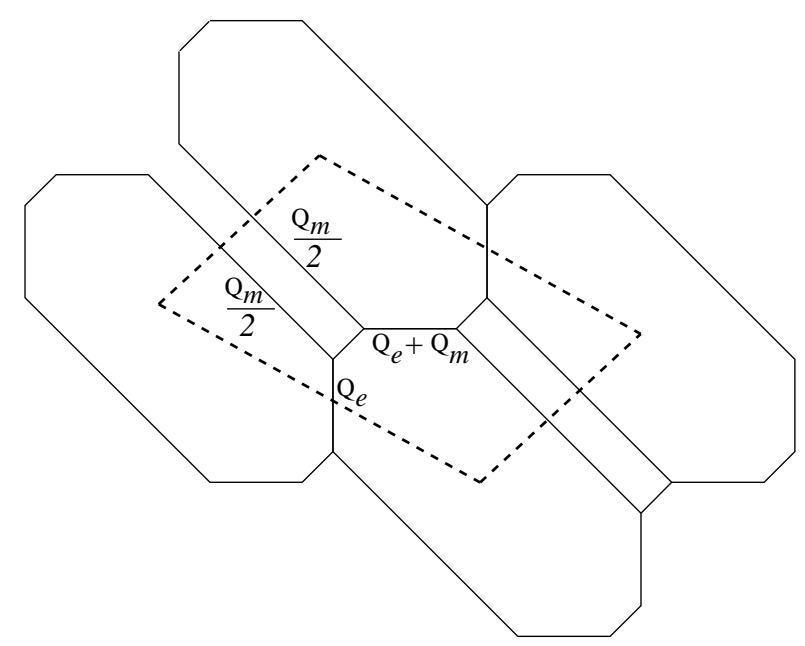

Figure 3: A quarter-BPS dyon carrying reducible charges $Q_{e}$ and $Q_{m}$ with $\operatorname{gcd}\left(Q_{e} \wedge Q_{m}\right)=2$

configurations. For example, a strand may split into two or more parallel strands, or the junction may open up into a triangle. Any complicated periodic network made out of strands with charges that are linear combinations of $Q_{e}$ and $Q_{m}$, and such that the total charge flowing across one side of the fundamental cell is $Q_{e}$, and through the other side $Q_{m}$ will give a possible realization of the dyon as shown in Fig. 3. If that is possible, the M-theory lift would predict a more complicated expression for the dyon degeneracies. For simplicity, in the following analysis we restrict to configurations with no momentum or KK charge.

To understand the relation between the value of $I$ and the possible variety of string webs that may describe a dyon with given charges, it is useful to consider a graph in the space of charges that is topologically dual to the string web. A dual graph is constructed as follows. For every face of the web associate a vertex in the dual graph. If two faces $A$ and $B$ in the web share an edge then the corresponding vertices $A^{\prime}$ and $B^{\prime}$ in the dual graph are connected by a vector that is equal in magnitude to the central charge of the edge but rotated by $\pi / 2$ in orientation compared to the edge. Recall that each edge in the string web carries a central charge and that the relative angles between the edges mimic the angles between the corresponding central charge vectors. A junction has three faces and three edges which maps to a triangle in the dual graph with three vertices and three edges. Charge conservation at each junction means that the vector sum of the three edge vectors is zero. This then guarantees that the sides of the dual triangle actually close, as their vector sum is zero. A string web constructed from a period array of junctions then corresponds to a triangulation in the dual graph.

Now, the vertices of the dual graph will sit at integral points of the charge lattice, on the plane defined by the vectors $Q_{e}$ and $Q_{m}$. The graph will have a fundamental cell with sides $Q_{e}$ and $Q_{m}$. Our invariant $I$ counts the number of integral points inside the fundamental cell. In 
this dual description, it is clear geometrically that $Q_{e}^{i} Q_{m}^{j}-Q_{e}^{j} Q_{m}^{i}$ are the various components of the area 2-form associated with the fundamental cell. If all the components do not have common factor then the fundamental parallelogram does not have any integral points either on the edges or inside 18 .

Let us see in more detail that $I$ counts the number of integral points inside the fundamental cell. If all $Q_{e}^{i} Q_{m}^{j}-Q_{e}^{j} Q_{m}^{i}$ are multiples of I, then consider any vector $Q$ such that $Q \cdot Q_{e}$ is not a multiple of $I$. If such a vector does not exist, then $Q_{e}$ is a multiple of $I$ and there are extra integral points on the edges of the parallelogram. If on the other hand, such a vector exists, then $\frac{Q \cdot Q_{e}}{I} Q_{m}^{i}-\frac{Q \cdot Q_{m}}{I} Q_{e}^{i}$ is an integral charge vector that is a linear combination of $Q_{e}$ and $Q_{m}$ with fractional coefficients. Up to shifts by $Q_{e}$ and $Q_{m}$ it will lie inside the parallelogram. Conversely, if the lattice of integral points that are coplanar with $Q_{e}$ and $Q_{m}$ has a smaller fundamental cell than the parallelogram with sides $Q_{e}$ and $Q_{m}$, then $Q_{e}=a Q_{1}+b Q_{2}$, $Q_{m}=c Q_{1}+d Q_{2}, a d-b c>1$. There will be $a d-b c$ points inside the parallelogram, and as $Q_{e} \wedge Q_{m}=(a d-b c) Q_{1} \wedge Q_{2}, I=a d-b c$ is the number of points inside the parallelogram with sides $Q_{e}$ and $Q_{m}$.

We thus see that if $I>1$, then the fundamental cell in the dual graph has an internal integral point. Each of the internal points can be used as a vertex for a triangulation. A generic periodic triangulation subdivides a fundamental cell into at most $2 I$ faces. ${ }^{1}$ In the dual description, a string web on the torus that carries charges $Q_{e}$ and $Q_{m}$ will have at most $2 I$ three-strand junctions, and $I$ faces.

To put it differently, note that $I=1$ without any internal faces is a genus two surface after M-theory lift. Adding a face increases the genus by one. Hence the genus of a M-theory lift of a string web with the invariant $I$ will be a surface with genus $I+1$.

When a face opens up at a string junction, its size is a zero mode in that the mass of dyon is independent of the size of the additional face. These zero modes and the invariant $I$ have been discussed earlier in a related context of quarter-BPS dyons in field theory using their realization as string junctions going between a collection D3-branes [19]. In that context, the zero mode is one of the collective coordinates that must be quantized to determine the ground state wavefunction. Similar comments might apply in our case. More work is need to obtain a definite interpretation of the higher genus contribution.

\section{States with Negative Discriminant}

An important test of the dyon degeneracy formula is that for large charges, the logarithm of the predicted degeneracy $\log d(Q)$ matches with Bekenstein-Hawking entropy. To make this

\footnotetext{
${ }^{1}$ This follows from Euler formula on the torus: a triangulation has $3 / 2$ edges for each face, hence the number of vertices is $1 / 2$ the number of faces.
} 
comparison, for a given a BPS dyonic state with electric and magnetic charges $\left(Q_{e}, Q_{m}\right)$, one would like to find a supersymmetric black hole solution of the effective action with the same charges and mass and then compute its entropy. It is useful to define the 'discriminant' $\Delta$ by

$$
\Delta(Q)=Q_{e}^{2} Q_{m}^{2}-\left(Q_{e} \cdot Q_{m}\right)^{2} .
$$

which is the unique quartic invariant of the full U-duality group $S O(22,6 ; \mathbb{Z}) \times S L(2, \mathbb{Z})$. For a black hole with charges $\left(Q_{e}, Q_{m}\right)$, the attractor value of the horizon area is proportional to the square root of the discriminant and the entropy is given by

$$
S(Q)=\pi \sqrt{\Delta(Q)} .
$$

On the microscopic side also, the discriminant is a natural quantity. It is useful to think of $S L(2, \mathbb{Z})$ as $S O(1,2 ; \mathbb{Z})$ which has a natural embedding into $S p(2, \mathbb{Z}) \sim S O(2,3 ; \mathbb{Z})$. The dyon degeneracy formula depends on the T-duality invariant vector of $S O(1,2 ; \mathbb{Z})$

$$
\left(\begin{array}{c}
Q_{m}^{2} / 2 \\
Q_{e}^{2} / 2 \\
Q_{e} \cdot Q_{m}
\end{array}\right)
$$

The discriminant is the norm of this vector with the Lorentzian metric

$$
\left(\begin{array}{ccc}
0 & 2 & 0 \\
2 & 0 & 0 \\
0 & 0 & -1
\end{array}\right) \text {. }
$$

With this norm, for a given state $\left(Q_{m}^{2} / 2, Q_{e}^{2} / 2, Q_{e} \cdot Q_{m}\right)$,the vector (5.3) is spacelike, lightlike, or timelike depending on whether $\Delta$ is positive, zero, or negative. We can accordingly refer to the state as spacelike, lightlike, or timelike.

Clearly, to obtain a physically sensible, nonsingular, supersymmetric, dyonic black hole solution in supergravity, it is necessary that the discriminant defined in (5.1) is positive and large so that the entropy defined in (5.2) is real. The vector in (5.3) in this case is spacelike. This fact seems to lead to the following puzzle regarding the dyon degeneracy formula. The formula predicts a large number of states that can have vanishing or negative discriminant. Since there are no big black holes in supergravity in that case, there does not appear to be a supergravity realization of these states predicted dyon degeneracy. This raises the following question. Do the lightlike and timelike states predicted by the dyon degeneracy formula actually exist in the spectrum and if so what is their macroscopic realization? It is important to address this question to determine the range of applicability of the dyon degeneracy formula. 


\subsection{Microscopic Prediction}

To start with, let us emphasize that the lightlike or timelike states are not necessarily pathological even though there is no supergravity solution corresponding to them. The simplest example of a lightlike state is the half-BPS purely electric state in the heterotic frame with winding $w$ along a circle and momentum $n$ along the same circle [20, 21]. For such a state, $Q_{e}^{2}=2 n w$ is nonzero but since it carries no magnetic charge, both $Q_{m}^{2}$ and $Q_{e} \cdot Q_{m}$ are zero and hence the discriminant is zero. The supergravity solution is singular but higher derivative corrections generate a horizon with the correct entropy [22, 23, 24]. We would like to know if similarly there exist quarter-BPS states that are timelike or lightlike in accordance with the predictions of the dyon degeneracy formula and what their supergravity realization is.

In general, it is not easy to extract closed form asymptotics from the degeneracy formula in this regime when the discriminant is negative or zero. But we have already encountered a simple example of a timelike state in $\oint_{3}$. The states with $\left(Q_{m}^{2} / 2, Q_{e}^{2} / 2, Q_{e} \cdot Q_{m}\right)$ equal to $(-1,-1, N)$ have discriminant $1-N^{2}$ which can be arbitrarily negative and we have determined the degeneracy of this state to be $d(-1,-1, N)=N$. Do such states exist in the physical spectrum, and if so what is their supergravity realization that can explain the degeneracy?

It is easy to construct such a state from a collection of winding, momentum, KK5, NS5 states in heterotic description. We choose a convenient representative that makes the supergravity analysis in the following section simpler. We consider heterotic string compactified on $\mathbf{T}^{\mathbf{4}} \times \mathbf{S}^{\mathbf{1}} \times \tilde{\mathbf{S}}^{\mathbf{1}}$. Let the winding and momentum around the circle $S^{1}$ be $w$ and $n$ and around the circle $\tilde{\mathbf{S}}^{\mathbf{1}}$ be $\tilde{w}$ and $\tilde{n}$. Similarly, $K$ and $W$ are the KK-monopole and NS5-brane charges associated with the circle $\mathbf{S}^{\mathbf{1}}$ whereas $\tilde{K}$ and $\tilde{W}$ are the KK-monopole and NS5-brane charges associated with the circle $\tilde{S}^{1}$. Note that the state with charge $W$ can be thought of as an NS5 brane wrapping along $\mathbf{T}^{\mathbf{4}} \times \tilde{\mathbf{S}}^{\mathbf{1}}$ whereas the states with charges $\tilde{W}$ is wrapping along $\mathbf{T}^{\mathbf{4}} \times \mathbf{S}^{\mathbf{1}}$. While the state that magnetically dual to $n$ is $K$ in terms of Dirac quantization condition, the state that is S-dual to $n$ is $W$. Similar comment holds for other states. With this notation, we then choose the charges $\Gamma=\left(Q_{e} \mid Q_{m}\right)=(n, w ; \tilde{n}, \tilde{w} \mid W, K ; \tilde{W}, \tilde{K})$ to be

$$
\Gamma=(1,-1 ; 0, N \mid 0,0 ; 1,-1) .
$$

This state clearly has $\left(Q_{m}^{2} / 2, Q_{e}^{2} / 2, Q_{e} \cdot Q_{m}\right)=(-1,-1, N)$. We will show in the appendix $\$ 5.2$ that the supergravity solution corresponding to this state with the required degeneracy has two centers instead of one. One center is purely electric with charge vector

$$
\Gamma_{1}=(1,-1 ; 0, N \mid 0,0 ; 0,0),
$$

and the other purely magnetic with charge vector

$$
\Gamma_{2}=(0,0 ; 0,0 \mid 0,0 ; 1,-1)
$$


both separated by a distance $L$. The corresponding supergravity solution exists for charge configuration with a positive, nonzero value for the distance $L$ both for positive and negative $N$ in a large regions of the moduli space but not for all values of the moduli. We discuss the explicit solution and as well as the moduli dependence and lines of marginal stability in the next subsections.

It is easy to see that such a two-centered solution has the desired degeneracy in agreement with the prediction from the dyon partition function. Each center individually contributes no entropy because for example the electric center by itself has $Q_{e}^{2} / 2=-1$ and hence carries no left-moving oscillations. However, because the charges are not mutually local, there is a net angular momentum $j=N / 2$ in the electromagnetic field. For large $N$, the angular momentum multiplet has $2 j+1$ or $N$ states in agreement with the dyon degeneracy formula. We thus see that at least some of the states with negative discriminant predicted by the dyon degeneracy formula can be realized physically but as multi-centered configurations.

\subsection{Supergravity Analysis}

For the supergravity analysis of the dyonic configurations, it is convenient to use the $\mathcal{N}=2$ special geometry formalism. Consider Type-II string compactified on a Calabi-Yau threefold with Hodge numbers $\left(h^{1,1}, h^{2,1}\right)$ which results in a $\mathcal{N}=2$ supergravity in four dimensions with

$h^{1,1}$ vector multiplets and $h^{2,1}+1$ hyper-multiplets. The hypermultiplets will not play any role in our analysis. The vector multiplet moduli space is a special Kähler geometry parameterized by $h^{1,1}+1$ complex projective coordinates $\left\{X^{I}\right\}$ with $I=0,1, \ldots, h^{1,1}$ and $\left\{\lambda X^{I}\right\} \sim\left\{X^{I}\right\}$. The low energy effective action of the vector multiplets is completely summarized by a prepotential which is a homogeneous function $F\left(X^{I}\right)$ of degree two,

$$
F\left(\lambda X^{I}\right)=\lambda^{2} F\left(X^{I}\right)
$$

In particular, the Kähler potential $\mathcal{K}$ is determined in terms of the prepotential by

$$
e^{-\mathcal{K}}=i\left(\bar{X}^{I} F_{I}-X^{I} \bar{F}_{I}\right)
$$

where

$$
F_{I}=\frac{\partial F}{\partial X^{I}}
$$

In our case, since we have a special Calabi-Yau $\mathbf{K} \mathbf{3} \times \mathbf{T}^{\mathbf{2}}$, we actually get $\mathcal{N}=4$ supersymmetry which has two additional gravitini multiplets. With our charge assignment, the vector fields in the gravitini multiplets are not excited and we can restrict our attention to the $\mathcal{N}=2$ sector. In the heterotic frame, we have excited electric and magnetic charges (5.5) which couple only to gauge fields associated with the $\mathbf{T}^{\mathbf{2}}$ part and to the metric and the dilaton-axion. As a result, the sigma model corresponding to the black hole configuration in $\mathbf{R}^{\mathbf{4}}$ is completely 
factorized into the $\mathbf{T}^{\mathbf{4}}$ conformal field theory and the sigma model involving $\mathbf{T}^{\mathbf{2}} \times \mathbf{R}^{\mathbf{4}}$ parts. This implies that for analyzing our charge configuration, we can restrict our attention to the moduli fields associated with $\mathbf{T}^{2}$ and the dilaton-axion. The prepotential in this case can be chosen to be

$$
F\left(X^{I}\right)=-\frac{X^{1} X^{2} X^{3}}{X^{0}}
$$

which corresponds to the so called STU model. Here

$$
S=X^{1} / X^{0}=a+i e^{-2 \Phi}
$$

is the dilaton-axion field, where $a$ is the axion and $\Phi$ is the dilaton in the heterotic frame. Similarly $T=X^{2} / X^{0}$ is the complex structure modulus of the $\mathbf{T}^{\mathbf{2}}$ and $U=X^{3} / X^{0}$ is the Kähler modulus of the $\mathbf{T}^{\mathbf{2}}$ in the heterotic frame. All other moduli fields do not vary in the geometry corresponding to our charge configuration. Restricting to the STU model greatly simplifies the analysis. Indeed this motivates the choice of the charges as in (5.5).

Given the prepotential (5.11) specifying the special geometry, there is a natural symplectic action $S p(4, \mathbb{R})$ on $\left(X^{I}, F_{I}\right)$. Similarly, the charges $\left(p^{I}, q_{I}\right)$ transform as a symplectic vector. These charges are more naturally defined in the Type-IIA frame, where $q_{I}$ are the electric charges arising from D0-brane and wrapped D2-branes, and $p^{I}$ are the magnetic charges arising from D6-brane and wrapped D4-branes.

A general supersymmetric multi-centered dyonic solution has a metric of the form

$$
-e^{-2 G(\vec{r})}\left(d t+\omega_{i} d x^{i}\right)^{2}+e^{2 G(\vec{r})}\left(d r^{2}+r^{2} d \Omega_{2}^{2}\right) .
$$

The four complex moduli fields $X^{I}$ that solve the equations of motion are determined in terms of the function $G$ and harmonic functions $H^{I}$ and $H_{I}$ by the eight real equations

$$
\begin{aligned}
e^{-G}\left(X^{I}-\bar{X}^{I}\right) & =H^{I}(\vec{r}) \\
e^{-G}\left(F_{I}-\bar{F}_{I}\right) & =H_{I}(\vec{r}),
\end{aligned}
$$

in the gauge

$$
e^{-\mathcal{K}}=\frac{1}{2}
$$

with the Kähler potential given by (5.9). For a configuration with $s$ charge centers with charges $\Gamma_{a}=\left(p_{a}^{I}, q_{I a}\right), a=1, \ldots s$ localized at the centers $\vec{r}=\vec{r}_{a}$ respectively, the harmonic functions $H_{I}$ and $H^{I}$ are given by [25]

$$
H^{I}=h^{I}+\sum_{a=1}^{s} \frac{p_{a}^{I}}{\left|\vec{r}-\vec{r}_{a}\right|}, \quad H_{I}=h_{I}+\sum_{a=1}^{s} \frac{q_{I a}}{\left|\vec{r}-\vec{r}_{a}\right|} .
$$


The constants of integration $h_{I}$ and $h^{I}$ will be determined in terms of the moduli fields shortly. Let $\Sigma(Q)$ be the entropy of the black hole which in our case equals $\pi \sqrt{\Delta(Q)}$. Then geometry of the solution is completely determined in terms of these harmonic functions [25]. The moduli are given by

$$
\frac{X^{A}}{X^{0}}=\frac{\partial_{A} \Sigma(H)-i H^{A}}{\partial_{0} \Sigma(H)-i H^{0}}
$$

with $A=1,2,3$ and $\partial_{A}=\partial / \partial H^{A}$. The metric is given by

$$
\begin{gathered}
e^{-2 G}=\Sigma(H), \\
\nabla \times \omega=H^{I} \nabla H_{I}-H_{I} \nabla H^{I} .
\end{gathered}
$$

Taking divergence of both sides then implies the Denef's constraint [26]

$$
H^{I} \nabla^{2} H_{I}-H_{I} \nabla^{2} H^{I}=0 .
$$

This is a consistency condition for a solution with $s$ centers to exist, where $\nabla^{2}$ is the flat space Laplacian in $\mathbf{R}^{3}$. This implies the following $s$ equations

$$
\left(h_{I} p_{a}^{I}-h^{I} q_{I a}\right)+\sum_{b=1}^{s} \frac{\left(p_{a}^{I} q_{I b}-q_{I a} p_{b}^{I}\right)}{\left|\vec{r}_{a}-\vec{r}_{b}\right|}=0,
$$

where sum over repeated $I$ index is assumed. Summing over the index $a$ in the equation above gives the summed constraint

$$
\left(h_{I} p^{I}-h^{I} q_{I}\right)=0,
$$

where $p^{I}=\sum p_{a}^{I}$ and $q_{I}=\sum q_{I a}$ are the total charges.

The values of the moduli fields $S=S_{1}+i S_{2}, T=T_{1}+i T_{2}$ and $U=U_{1}+i U_{2}$ at asymptotic infinity are specified by six real constants. The solutions on the other hand are determined by eight real constants of integration $\left(h^{I}, h_{I}\right), I=0,1,2,3$ which however must satisfy two real constraints (5.16) and (5.23). Thus, they can be determined in terms of the six asymptotic values of the moduli fields and the complete supersymmetric solution for all fields is then determined by (5.14), (5.13), and (5.17).

Specializing to our case, we will consider a two-centered solution so $s=1,2$. We restrict ourselves to a region of moduli space where $\mathbf{T}^{2}$ is factorized into two circles $\mathbf{S}^{1} \times \tilde{\mathbf{S}}^{1}$ and there is no $B$ field on the torus. In other words, we work on the submanifold of the moduli space with $T_{1}=U_{1}=0$. Let $R_{1}$ and $R_{2}$ be the radii of the circles $\mathbf{S}^{1}$ and $\tilde{\mathbf{S}}^{1}$ respectively, $\chi$ be the asymptotic expectation value of the axion, and $g^{2}$ be the string coupling given by the asymptotic value of $e^{2 \Phi}$. A nonzero value of $\chi$ will be essential to obtain a well defined solution. Given this asymptotic data

$$
S_{\infty}=\chi+\frac{i}{g^{2}}, \quad T_{\infty}=i \frac{R_{1}}{R_{2}}, \quad U_{\infty}=i R_{1} R_{2},
$$


we now proceed to determine the constants of integration $\left(h^{I}, h_{I}\right)$.

At asymptotic infinity, $G(\vec{r})$ vanishes, so the solutions (5.14) reduce to

$$
2 \operatorname{Im}\left(X^{I}\right)=h^{I}, \quad 2 \operatorname{Im}\left(F_{I}\right)=h_{I} .
$$

Let $X_{\infty}^{0}=\alpha+i \beta$. Then from (5.14) and (5.24) we see that the constants of integration are given by

$$
\begin{array}{ll}
h^{0}=2 \beta & h_{0}=-2 \alpha \frac{R_{1}^{2}}{g^{2}}-2 R_{1}^{2} \beta \chi \\
h^{1}=2 \alpha \frac{1}{g^{2}}+2 \beta \chi & h_{1}=2 \beta R_{1}^{2} \\
h^{2}=2 \alpha \frac{R_{1}}{R_{2}} & h_{2}=2 \beta \frac{R_{1} R_{2}}{g^{2}}-2 \alpha \chi R_{1} R_{2} \\
h^{3}=2 \alpha R_{1} R_{2} & h_{3}=2 \beta \frac{R_{1}}{R_{2} g^{2}}-2 \alpha \chi \frac{R_{1}}{R_{2}} .
\end{array}
$$

The two constants $\alpha$ and $\beta$ that we have introduced are in turn determined in terms of the charges by plugging (5.26) into the two constraint equations (5.16) and (5.23). Equation (5.16) in particular implies

$$
\left|X^{0}\right|^{2}=\alpha^{2}+\beta^{2}=\frac{1}{16 S_{2} T_{2} U_{2}}=\frac{g^{2}}{16 R_{1}^{2}} .
$$

\subsection{Moduli Dependence and Lines of Marginal Stability}

So far our analysis is valid for any charge assignment but with the specific choice of the asymptotic moduli as in (5.24). The remaining equations (5.23) as well as (5.26) depend on the specific charge assignment of the configuration under study. To use the attractor equations to analyze the geometry for our charge configuration (5.6) and (5.7), we first translate the charges given in the heterotic frame to purely D-brane charges in the Type-IIA frame. The charges $\left(p^{I}, q_{I}\right)$ in the Type-IIA arise from various D-branes wrapping even-cycles. We label charges so that $q_{0}$ is the number of D0-branes, $q_{1}$ is the number of D2-branes wrapping the $\mathbf{T}^{2}, q_{2}$ is the number of D2-branes wrapping a 2-cycle $\Sigma_{2}$ in $\mathbf{K} 3, q_{3}$ is the number of D2-branes wrapping a 2-cycle $\tilde{\Sigma}_{2}$ that has intersection number one with the cycle $\Sigma_{2}$. Similarly, $p^{0}$ is the number of D6-branes wrapping $\mathbf{K} 3 \times \mathbf{T}^{2}, p^{1}, p^{2}, p^{3}$ are the number of D4-branes wrapping $\mathbf{K} 3, \tilde{\Sigma}_{2} \times \mathbf{T}^{\mathbf{2}}$ and $\Sigma_{2} \times \mathbf{T}^{2}$ respectively. By the duality chain in appendix B, these charges in the Type-IIA frame are related to the electric and magnetic charges $\left(Q_{e}, Q_{m}\right)$ in the heterotic frame by

$$
\begin{gathered}
Q_{e}=(n, w ; \tilde{n}, \tilde{w}) \equiv\left(q_{0},-p^{1}, q_{2}, q_{3}\right) \\
Q_{m}=(W, K ; \tilde{W}, \tilde{K}) \equiv\left(q_{1}, p^{0}, p^{3}, p^{2}\right) .
\end{gathered}
$$


Now we are ready to apply the $\mathcal{N}=2$ formalism to our two-centered configuration with the charge assignment (5.6) and (5.7). The electric center has charges

$$
\Gamma_{1}=(1,-1,0, N \mid, 0,0,0,0)
$$

and the magnetic center has charges

$$
\Gamma_{2}=(0,0,0,0 \mid 0,0,1,-1)
$$

The constraint (5.23) then reads

$$
h_{1}-h^{0}-N h^{3}+h_{3}-h_{2}=0 .
$$

Substituting the values of the integration constants $h^{I}$ and $h_{I}$ in terms of $\alpha$ and $\beta$ from (5.26) into this equation, we obtain one equation for the two unknowns $\alpha$ and $\beta$ in terms of charges and asymptotic moduli

$$
\beta\left(R_{1}^{2}-1+\frac{R_{1}}{R_{2} g^{2}}\left(1-R_{2}^{2}\right)\right)+\alpha\left(-N R_{1} R_{2}-\chi \frac{R_{1}}{R_{2}}+\chi R_{1} R_{2}\right)=0
$$

Combining this with the second equation (5.30) that comes from the gauge fixing constraint $e^{-\mathcal{K}}=\frac{1}{2}$ (5.16), we can now solve for the two unknowns to obtain

$$
\alpha=\frac{\left(R_{1}^{2}-1+\frac{R_{1}}{R_{2} g^{2}}\left(1-R_{2}^{2}\right)\right) g}{4 R_{1}\left(N R_{1} R_{2}+\chi \frac{R_{1}}{R_{2}}-\chi R_{1} R_{2}\right) \Lambda}, \quad \beta=\frac{g}{4 R_{1} \Lambda},
$$

where

$$
\Lambda^{2}=1+\left(\frac{R_{1}^{2}-1+\frac{R_{1}}{R_{2} g^{2}}\left(1-R_{2}^{2}\right)}{-N R_{1} R_{2}-\chi \frac{R_{1}}{R_{2}}+\chi R_{1} R_{2}}\right)^{2} .
$$

We have thus determined the integrations constants (5.26) that appear in the solution (5.17) completely in terms of the asymptotic moduli and the charges. The geometry of the solution is in tern determined entirely in terms of the harmonic functions. In particular the separation $L$ between the two centers can be obtained by solving Denef's constraint (5.22), which for our configuration becomes

$$
h_{2}-h_{3}=\frac{N}{L}
$$

we have,

$$
2 \frac{R_{1}}{R_{2}}\left(R_{2}^{2}-1\right)\left(\frac{\beta}{g^{2}}-\alpha \chi\right)=\frac{N}{L}
$$

Since $L$ is the separation between the two centers, it must be positive. This requires that $\left(\frac{\beta}{g^{2}}-\alpha \chi\right)$ must be positive. It is clear that this can be ensured for a large region of moduli 
space. The locus in the moduli space where this quantity becomes negative determines the line of marginal stability in the upper half $S$ plane by the equation

$$
\frac{1}{g^{2}}-\left(\frac{R_{1}^{2}-1+\frac{R_{1}}{R_{2} g^{2}}\left(1-R_{2}^{2}\right)}{N R_{1} R_{2}+\chi \frac{R_{1}}{R_{2}}-\chi R_{1} R_{2}}\right) \chi=0,
$$

which simplifies to

$$
\chi=N \frac{R_{1} R_{2}}{R_{1}^{2}-1} \frac{1}{g^{2}} .
$$

This equation defines a straight line in the complex $S_{\infty}$ plane with $S_{\infty}=\chi+i / g^{2}$. Note that the slope of the line is proportional to $N$. For fixed $R_{1}$ and $R_{2}$, this defines a curve of marginal stability in the complex $S_{\infty}$ plane. For positive $N$, the desired two-centered solution exists if $\chi+i / g^{2}$ lies to the left of the line defined by the equation (5.42). In this region, the distance between the two centers determined by Denef's constraint (5.40) is positive and finite. After crossing the line of marginal stability, the solution ceases to exist because then there is no solution with positive $L$ to the constraint (5.40). As one approaches the line of marginal stability from the left, the distance $L$ between the electric and magnetic centers goes to infinity. In other words, the total state with charge vector $\Gamma$ decays into two fragments with charge vectors $\Gamma_{1}$ and $\Gamma_{2}$. The mass $M$ of the state with charge $\Gamma$ is given in terms of the central charge by the BPS formula $M=|Z(\Gamma)|$ with

$$
Z=e^{\mathcal{K} / 2}\left(p^{I} F_{I}-q_{I} X^{I}\right)
$$

At the curve of marginal stability, it is easy to check that $Z(\Gamma)=Z\left(\Gamma_{1}\right)+Z\left(\Gamma_{2}\right)$. Hence the state with charge vector $\Gamma$ can decay into its fragments with charge vectors $\Gamma_{1}$ and $\Gamma_{2}$ by a process that is marginally allowed by the energetics and charge conservation.

Similarly, if $N$ is negative, the straight line defined by (5.3) has negative slope and a solution with positive $L$ exists only to the right of this line. As we have noted, the S-transformation maps the configuration with $N$ positive to $N$ negative. Hence the line with positive slope gets mapped to a line with negative slope and thus the curves of marginal stability move under S-duality. The fact that a two centered solution exists for both signs and with the correct degeneracy is consistent with our prescription for extracting S-duality invariant spectrum proposed in $\S 3$. In the wedge between the two lines defined the two lines of marginal stability for $N$ positive and $N$ negative, both states coexist. In other regions, only one or the other state exists.

The simplicity of the line of marginal stability defined by (5.42) has a simple and beautiful interpretation from the string web picture reviewed in $\$$. Indeed a string web made out of strands with certain charges exists only if these charges can be carried by a supersymmetric string in six dimensions. If one crosses a line of degeneration in the moduli space, across which a strand with charges, say, $Q_{e}+Q_{m}$ shrinks to zero length and is replaced by a strand with charge 
$Q_{e}-Q_{m}$, the quarter-BPS state will decay if no supersymmetric string with charge $Q_{e}-Q_{m}$ exists. The line of degeneration is simply the line at which a string of charge $Q_{e}$ along one cycle of the torus and a string of charge $Q_{m}$ along the other can be simultaneously supersymmetric. This is equivalent to the requirement that the phase of $S$ is the same as the angle between the central charge vectors for $Q_{e}$ and $Q_{m}$, that defines a straight line in the $S$ plane. In the present case $Q_{e}=(1,-1,0, N)$ and $Q_{m}=(0,0,1,-1)$, hence $Q_{e} \pm Q_{m}=(1,-1, \pm 1, N \mp 1)$. $\frac{1}{2}\left(Q_{e} \pm Q_{m}\right)^{2}=-1 \pm N$, but a BPS string with charge $Q$ must have $Q^{2} / 2 \geq-1$. Hence the line of degeneration of the string web is indeed a line of marginal stability.

It is not surprising that the existence of quarter-BPS dyons depends on the moduli and that there are lines of marginal stability which separate the regions where the state exists from where it does not exist. This phenomenon is well known in the field theory context [27]. Moduli dependence of the spectrum of quarter-BPS dyons and the lines of marginal stability in the present string-theoretic context have been observed and analyzed from a different perspective also in the forthcoming publication [28].

\section{Interpretation and Discussion}

As we have seen, the interpretation of the proposed dyon degeneracy formula presents many subtleties. It is unlikely that the formula is valid in all regions of moduli space for all charges in a way envisioned in [1, 2] that depends only on the three invariants $Q_{e}^{2} / 2, Q_{m}^{2} / 2$, and $Q_{e} \cdot Q_{m}$. We summarize below our observations and what we believe would be the consistent physical interpretation of the dyon degeneracy formula.

- It is clear that the three invariants $\left(Q_{e}^{2} / 2, Q_{m}^{2} / 2, Q_{e} \cdot Q_{m}\right)$ do not uniquely specify the state and the degeneracy will depend on additional data. This is natural because the arithmetic duality group has many more invariants than the continuous duality group. We have identified a particular invariant $I$ which determines when the genus-two partition function is adequate but this is not the end of the story. To illustrate this point, let us consider an even more striking example of a quarter-BPS lightlike state for which additional data is required to specify the degeneracy of states. ${ }^{2}$ Consider a perturbative BPS state that is purely electric in the Type-IIA frame carrying winding $w$ along a circle of the $\mathbf{T}^{\mathbf{2}}$ factor and momentum $n$ along the same circle. In the heterotic frame it corresponds to a state with $w$ NS5-branes wrapping $\mathbf{T}^{4} \times \mathbf{S}^{\mathbf{1}}$ with momentum $n$ along the $\mathbf{S}^{1}$. For nonzero $n$ and $w$ the state carries arbitrary left-moving oscillations $N_{L}=n w$ and has entropy $2 \pi \sqrt{2} \sqrt{n w}$. Unlike a similar heterotic electric state which is half-BPS, these states are quarter-BPS because both right and left movers carry supersymmetry for the Type-II string. Now, for all such states, all three invariants $\left(Q_{e}^{2} / 2, Q_{m}^{2} / 2, Q_{e} \cdot Q_{m}\right)$ vanish and so

\footnotetext{
${ }^{2}$ We thank Boris Pioline for discussions on this point.
} 
does the discriminant. Thus there is a large set of legitimate quarter-BPS states with the same values for the three invariant, namely zero, but very different entropy depending on the values of $n$ and $w$. The degeneracy of such states cannot possibly be captured by the genus-two partition function. This example illustrates that additional data might be required to determine the degeneracy of states, although alternative explanations are possible. The difference might also be attributed to a difference between the absolute degeneracy of states and the supersymmetry index computed by the dyon degeneracy formula.

- The states with negative discriminant appear problematic at first because there is no black hole corresponding to them. We have seen that they can nevertheless have a sensible physical realization. In the specific example considered here the states are described as a two-centered configuration in supergravity. These configuration have the right degeneracy coming from the angular momentum multiplicity consistent with the prediction of the dyon degeneracy formula. We would like to propose that other negative discriminant states also exist and can be realized as complicated multi-centered configurations. The supergravity analysis also indicates that existence of these states is moduli dependent. The states exist over a large region of the moduli space but cannot exist in certain regions of the moduli space because the distance between the two centers determined by Denef's constraint goes to infinity. This shows that generically there are walls of marginal stability in the moduli space that separate regions where the states exist from regions where they do not. This is not surprising since even in field theory, quarter-BPS states in $\mathcal{N}=4$ theories are known to have curves of marginal stability [27, 29]. It is possible that this moduli dependence is related to the need to change the choice of contour to obtain an S-duality invariant answer. As these lines of marginal stability have a simple description in the string web picture, it might be possible to understand the change of contour from the M-theory lift of the string web.

- Despite these subtleties, it is also true that the dyon partition function has been derived from various points of views for specific charge configurations and in specific regions of moduli space. Considering the caveats above, a conservative interpretation of these results in our view is that the dyon degeneracy formula given in terms of the genus-two Siegel modular forms is exact and valid for specific charges in the specific regions of moduli spaces as well as for all charges related by a duality transformations in the dual regions of the moduli space. This already contains highly nontrivial information about the degeneracies of quarter-BPS bound states of various branes in the theory. This can be seen quite generally from the point of view of the string web picture. For a given charge configuration, and in a given region of the moduli space, if a string web is stable 
and can be lifted to a wrapped K3-wrapped M5-brane with a genus-two world sheet, then one can derive the degeneracy from the genus-two partition function of the left-moving heterotic string as has been done in [9, 5, 10]. However, as one moves around in the moduli space, the string web can become unstable. Once the string web is unstable, the dyon degeneracies can no longer be obtained from the genus-two partition function. Thus the derivation of the dyon partition function is valid in only a certain region of the moduli space for a given charge configuration. Moreover, for some quarter-BPS state, it may not be possible at all to represent the state as a string web that lifts to a K3wrapped M5-brane. For example, the Type-II perturbative states discussed above lift to a circle-wrapped M2-brane with genus-one topology and not to a K3-wrapped M5-brane with genus-two topology. A circle-wrapped M2-brane is nothing but the Type-II string and hence for these states the counting is correctly done using the genus-one partition function of the Type-II string and not using a genus-two partition function of the heterotic string. These examples clearly delineate the range of applicability of the dyon degeneracy formula.

\section{Acknowledgements}

It is a pleasure to thank Oren Bergman, K. Narayan, Boris Pioline, Greg Moore, and Ashoke Sen for valuable discussions. A. D. would like to acknowledge the hospitality of the Aspen Center for Physics and the theory group at the ASICTP where part of this work was completed.

\section{References}

[1] R. Dijkgraaf, E. P. Verlinde, and H. L. Verlinde, Counting dyons in $n=4$ string theory, Nucl. Phys. B484 (1997) 543-561, [hep-th/9607026].

[2] D. P. Jatkar and A. Sen, Dyon spectrum in chl models, hep-th/0510147.

[3] D. Gaiotto, A. Strominger, and X. Yin, New connections between $4 d$ and $5 d$ black holes, hep-th/0503217.

[4] D. Shih, A. Strominger, and X. Yin, Recounting dyons in $n=4$ string theory, hep-th/0505094.

[5] A. Dabholkar and S. Nampuri, Spectrum of dyons and black holes in chl orbifolds using borcherds lift, hep-th/0603066.

[6] J. R. David, D. P. Jatkar, and A. Sen, Product representation of dyon partition function in chl models, hep-th/0602254.

[7] J. R. David and A. Sen, Chl dyons and statistical entropy function from d1-d5 system, hep-th/0605210. 
[8] J. R. David, D. P. Jatkar, and A. Sen, Dyon spectrum in generic $n=4$ supersymmetric $z(n)$ orbifolds, hep-th/0609109.

[9] D. Gaiotto, Re-recounting dyons in $n=4$ string theory, hep-th/0506249.

[10] A. Dabholkar and D. Gaiotto, Spectrum of chl dyons from genus-two partition function, hep-th/0612011.

[11] G. Lopes Cardoso, B. de Wit, J. Kappeli, and T. Mohaupt, Asymptotic degeneracy of dyonic $n$ $=4$ string states and black hole entropy, JHEP 12 (2004) 075, [hep-th/0412287].

[12] G. Lopes Cardoso, B. de Wit, J. Kappeli, and T. Mohaupt, Black hole partition functions and duality, JHEP 03 (2006) 074, [hep-th/0601108].

[13] D. Shih and X. Yin, Exact black hole degeneracies and the topological string, JHEP 04 (2006) 034, [hep-th/0508174].

[14] J. H. Schwarz, Lectures on superstring and $m$ theory dualities, Nucl. Phys. Proc. Suppl. 55B (1997) 1-32, [hep-th/9607201].

[15] O. Aharony, A. Hanany, and B. Kol, Webs of $(p, q)$ 5-branes, five dimensional field theories and grid diagrams, JHEP 01 (1998) 002, [hep-th/9710116].

[16] K. Dasgupta and S. Mukhi, Bps nature of 3-string junctions, Phys. Lett. B423 (1998) 261-264, [hep-th/9711094].

[17] A. Sen, String network, JHEP 03 (1998) 005, [hep-th/9711130].

[18] E. P. Verlinde and M. Vonk, String networks and supersheets, hep-th/0301028.

[19] O. Bergman and B. Kol, String webs and 1/4 bps monopoles, Nucl. Phys. B536 (1998) 149-174, [hep-th/9804160].

[20] A. Dabholkar and J. A. Harvey, Nonrenormalization of the superstring tension, Phys. Rev. Lett. 63 (1989) 478.

[21] A. Dabholkar, G. W. Gibbons, J. A. Harvey, and F. Ruiz Ruiz, Superstrings and solitons, Nucl. Phys. B340 (1990) 33-55.

[22] A. Sen, Extremal black holes and elementary string states, Mod. Phys. Lett. A10 (1995) 2081-2094, [hep-th/9504147].

[23] A. Dabholkar, Exact counting of black hole microstates, Phys. Rev. Lett. 94 (2005) 241301, [hep-th/0409148].

[24] A. Dabholkar, R. Kallosh, and A. Maloney, A stringy cloak for a classical singularity, JHEP 12 (2004) 059, [hep-th/0410076]. 
[25] B. Bates and F. Denef, Exact solutions for supersymmetric stationary black hole composites, hep-th/0304094.

[26] F. Denef, Supergravity flows and d-brane stability, JHEP 08 (2000) 050, [hep-th/0005049].

[27] O. Bergman, Three-pronged strings and 1/4 bps states in $n=4$ super-yang-mills theory, Nucl. Phys. B525 (1998) 104-116, [hep-th/9712211].

[28] A. Sen, private communication and to appear, .

[29] P. C. Argyres and K. Narayan, String webs and the decay of supersymmetric particles, Int. J. Mod. Phys. A16S1C (2001) 962-966, [hep-th/0101139]. 